\title{
Sleep apnoea in women
}

\author{
Natalie Edwards, Ian Wilcox, Colin E Sullivan
}

Until recently the occurrence of sleep disordered breathing in premenopausal women has been considered to be so low as to be insignificant and, as a result, research into the consequent cardiovascular risk in this group has been completely neglected. However, recent epidemiological evidence based on sleep clinic data suggests that the incidence of significant sleep disordered breathing in young women (30-49 years) is as high as $4.9 \%$ (fig 1). ${ }^{1}$ Furthermore, while morbidity of women in the general population is lower than in men, recent epidemiological evidence suggests that five year survival is significantly poorer in women with obstructive sleep apnoea (OSA) than in a similar population of men. ${ }^{2}$ Thus, assessment of factors which may contribute to poorer outcomes in these women is important.

Cardiovascular control in men with OSA

Men with OSA have an excess of known cardiovascular risk factors such as hypertension, obesity (particularly central obesity), and increased insulin resistance. ${ }^{3}$ There is increasing evidence to suggest that OSA interacts adversely with these factors. This interaction occurs during both wakefulness and sleep. Epidemiological evidence assembled in a community based population study in the USA suggests that, after adjusting for independent risk factors associated with hypertension, OSA was independently responsible for up to a fivefold increase in the incidence of hypertension. ${ }^{4}$ Furthermore, Carlson and coworkers ${ }^{5}$ suggest that, while OSA is an independent risk factor for hypertension, it also has an additive effect when combined with age and obesity. Thus, blood pressure control is seriously compromised in men as a result of the presence of OSA. Similar potential risks have not been investigated in women with obstructive sleep apnoea.

David Read
Laboratory,
Department of
Medicine, University
of Sydney, NSW 2006,
Australia
N Edwards
I Wilcox
C E Sullivan
Correspondence to:
Dr N Edwards.

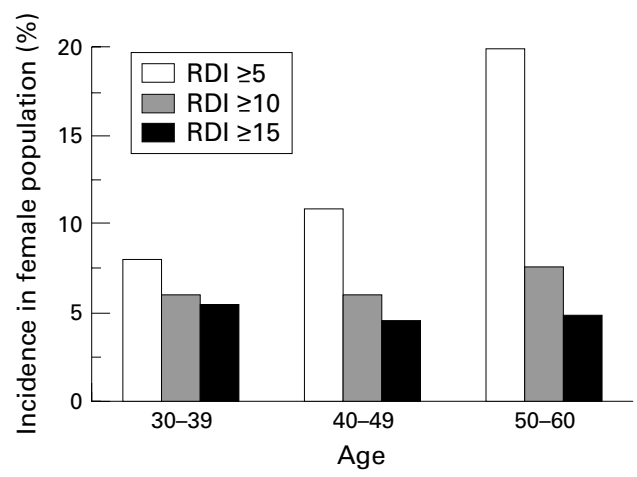

Figure 1 Age specific estimates of sleep disordered breathing in women. Contrary to many reports, these data suggest that the incidence of significant sleep disordered breathing in young women (most of whom will be premenopausal) is a significant public health issue. Modified from Young et al. ${ }^{1}$
During sleep, men with OSA have repetitive obstructive respiratory events which are associated with characteristic pathophysiological changes in ventilation and haemodynamics. Fluctuations in haemodynamic parameters which accompany obstructive apnoea during sleep in men include initial bradycardia (vagally mediated) followed by tachycardia associated with arousal at apnoea termination. ${ }^{6}$ Similarly, systemic blood pressure decreases towards the conclusion of the apnoea followed by a surge in both systolic and diastolic pressure associated with arousal occurring at apnoea termination. Surges in sympathetic nerve activity are believed to be predominantly responsible for haemodynamic fluctuations which are associated with obstructive respiratory events during sleep..$^{7-9}$ These changes in sympathetic nerve activity during obstructive events in sleep result from a number of factors including blood gas fluctuations (hypoxia and hypercapnia), ${ }^{10}$ large dynamic swings in intrathoracic pressure, ${ }^{11}{ }^{12}$ and arousals from sleep. ${ }^{13}$ While the result of these is an increased sympathetic tone overnight, increased sympathetic tone persists into the daytime in men with OSA. ${ }^{14}{ }^{15}$ Daytime sympathetic tone in these patients is reduced to normal levels once effective treatment with nasal continuous positive airways pressure (CPAP) has been established. ${ }^{16}$

In men there is some evidence that OSA increases the risk of vascular events and increases mortality independently of other known risk factors. ${ }^{3}{ }^{17} 18$ Haemodynamic fluctuations associated with obstructive events during sleep may contribute to the increased vascular risk in these men. Haemodynamics during obstructive apnoeas occurring in sleep have not to date been specifically investigated in either premenopausal or postmenopausal women.

\section{Ventilatory control during the menstrual cycle}

Until recently sleep disordered breathing in premenopausal women was believed to be a very rare occurrence. One of the predominant reasons cited for the supposed decreased incidence of sleep disordered breathing in premenopausal women was that they have greater central chemoreceptor drive, especially during the luteal phase of the menstrual cycle. ${ }^{19}{ }^{20}$ It is well described that minute ventilation increases during the luteal phase of the menstrual cycle and, further, that ventilatory responsiveness to hypercapnia is increased during this phase. ${ }^{21-23}$ Furthermore, basal minute ventilation and ventilatory responsiveness to hypercapnia are both potentiated in men receiving synthetic progesterone (medroxyprogesterone acetate).$^{24}$ Thus, increased ventilatory responsiveness during the luteal phase of the 
A
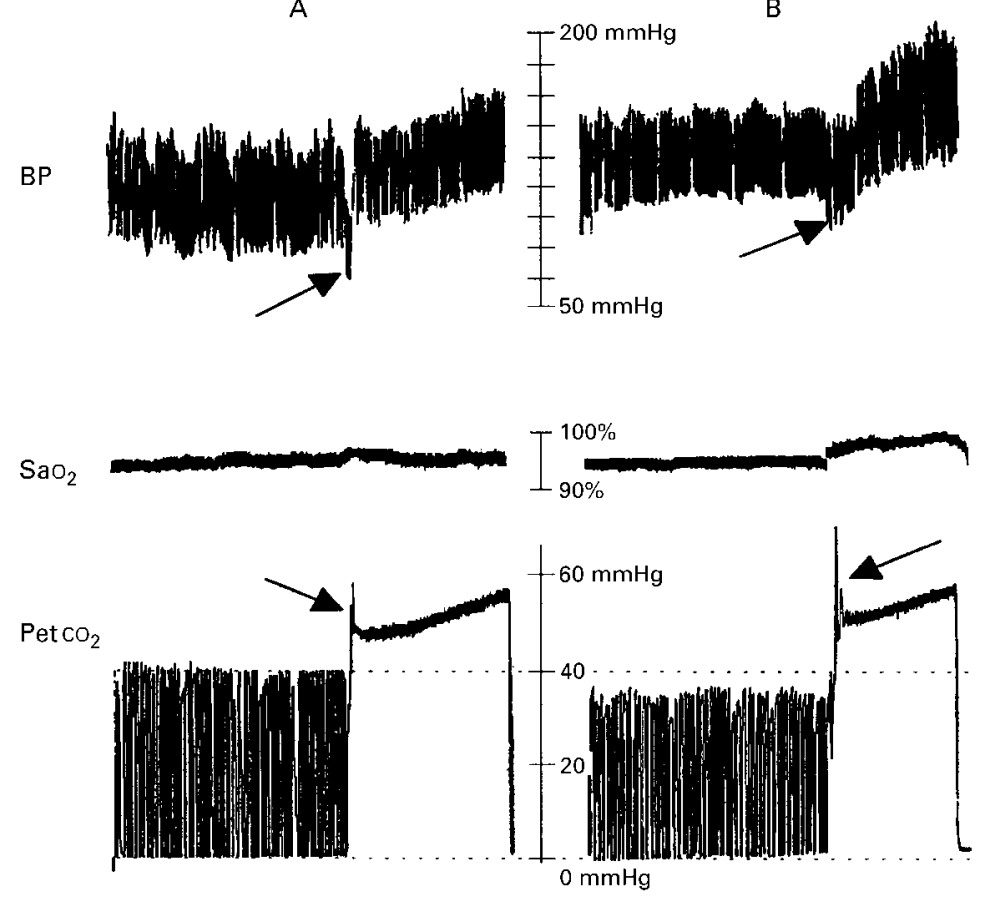

$\mathrm{Vt}$
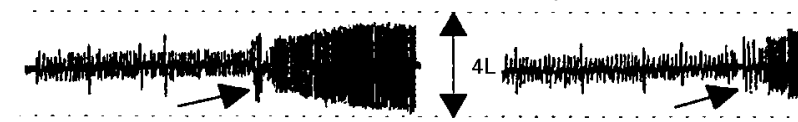

Figure 2 Cardiorespiratory responses to acute hyperoxic hypercapnia during (A) follicular and (B) luteal phase of the cycle. This study clearly shows an enhanced pressor response during the luteal phase of the menstrual cycle. The arrows on the figure indicate the beginning of hypercapnia after 5 min of normal tidal breathing.

cycle is specifically modified by changes in serum progesterone concentrations.

While ventilation and central chemoreceptor drive have been extensively studied throughout the normal menstrual cycle during wakefulness, little attention has been focused on menstrual cycle related breathing parameters during sleep. In women without significant sleep disordered breathing (having an apnoea/hypopnoea index of $<5 /$ hour of sleep), the incidence of apnoea or hypopnoea over the entirety of the night is unchanged by the phase of the cycle. ${ }^{25}$ During the transition from wakefulness to sleep a decrease in central chemoreceptor drive occurs such that, at sleep onset, central apnoea is common in healthy subjects with a consequent rise in end tidal carbon dioxide $\left(\mathrm{CO}_{2}\right)$ pressure of $0.3-1.1 \mathrm{kPa}^{26}$ The decrease in central chemoreceptor drive at sleep onset, as indicated by the incidence of central apnoea, and the rise in end tidal $\mathrm{CO}_{2}$ pressure are not influenced by hormonal fluctuations associated with the normal menstrual cycle. ${ }^{27}$ The hypoxic ventilatory response during sleep tends to be higher during the luteal phase of the menstrual cycle. ${ }^{28}$

Cardiovascular and haemodynamic changes associated with the menstrual cycle

Generally, the menstrual cycle is protective against cardiovascular disease. This is, however, primarily a result of blood composition which, as a result of high levels of serum oestradiol, tends towards lower levels of low density lipoproteins (LDL) and relatively higher levels

of high density lipoproteins (HDL), ${ }^{29}$ both of which protect against atherosclerotic plaque formation in blood vessels. There is also some suggestion that oestradiol plays a role in attenuating the endothelial mediated vasoconstrictor responses to substances such as angiotensin, especially in such high concentrations as are present during pregnancy. ${ }^{30} 31$ Thus, haemodynamics have little effect in the premenopausal protection against cardiovascular disease. In healthy women at rest there are conflicting reports in the literature concerning the status of haemodynamics, particularly the daytime blood pressure at rest; however, most studies which have included measurement of serum hormone concentrations in the experimental protocol suggest that blood pressure at rest does not vary significantly over the course of the menstrual cycle in normotensive women. ${ }^{21}{ }^{32-36}$

While blood pressure at rest appears uninfluenced by the stage of the menstrual cycle, in conditions of physical stress (cold stress and isometric exercise) blood pressure responses are potentiated during the luteal phase of the menstrual cycle. ${ }^{37}$ Furthermore, blood pressure responses to hyperoxic hypercapnia are significantly increased during the luteal phase of the cycle (fig 2). ${ }^{21}$ The influence of the cycle on blood pressure during hypercapnia is so strong that, in ovulatory cycles, the pressor response (defined as the increase in blood pressure for each $\mathrm{kPa}$ increase in end tidal $\mathrm{CO}_{2}$ ) is always higher during the luteal phase of the ovulatory cycle than it is during the follicular phase. ${ }^{21}$ Since no influence of the phase of the menstrual cycle was shown on hypercapnic heart rate responses in this study, the predominant haemodynamic mechanism responsible for the potentiated hypercapnic pressor response during the luteal phase of the cycle is thought to be an increased peripheral vascular resistance response. Current literature suggests that the autonomic nervous system (and specifically the sympathetic nervous system) is predominantly responsible for the enhanced pressor response to physical stress. While the actual output of the sympathetic nervous system either remains unchanged or is slightly decreased by the presence of increased serum progesterone concentrations (both during the normal menstrual $\operatorname{cycle}^{38}$ and in men receiving exogenous progesterone ${ }^{39}$ ), sympathetic responses (sweating, cardiovascular toxicity of cocaine) are enhanced in the presence of increased serum progesterone concentrations. ${ }^{38-40}$

While it is generally suggested that postmenopausal women have a significantly greater risk of developing OSA than premenopausal women, only one study has investigated the influence of hormone replacement therapy (HRT) on severity of obstructive apnoea in this population. $^{41}$ A slight improvement in the severity of apnoea in REM sleep only was found after administration of HRT.

In premenopausal women with OSA there is no evidence of fluctuation in indices of severity of sleep disordered breathing (respiratory disturbance index (RDI), apnoea length and decrease in arterial oxyhaemoglobin saturation $\left(\mathrm{SaO}_{2}\right)$ ) throughout the menstrual cycle during 

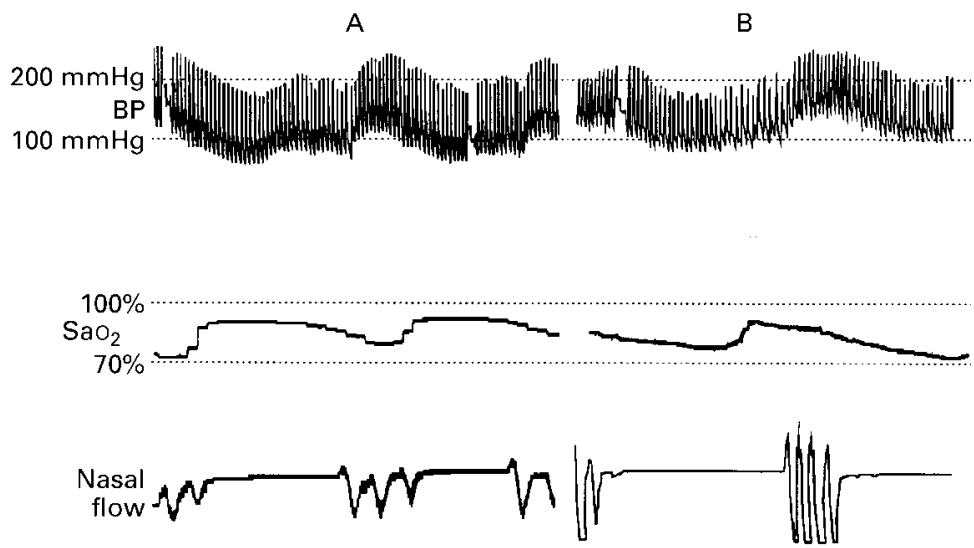

Figure 3 Haemodynamic consequences of apnoea during sleep in (A) a man and (B) a woman. The haemodynamic responses to apnoea include a decrease in blood pressure and heart rate during the course of the apnoea termination followed by a surge in both during the hyperpnoeic phase following apnoea termination.
A

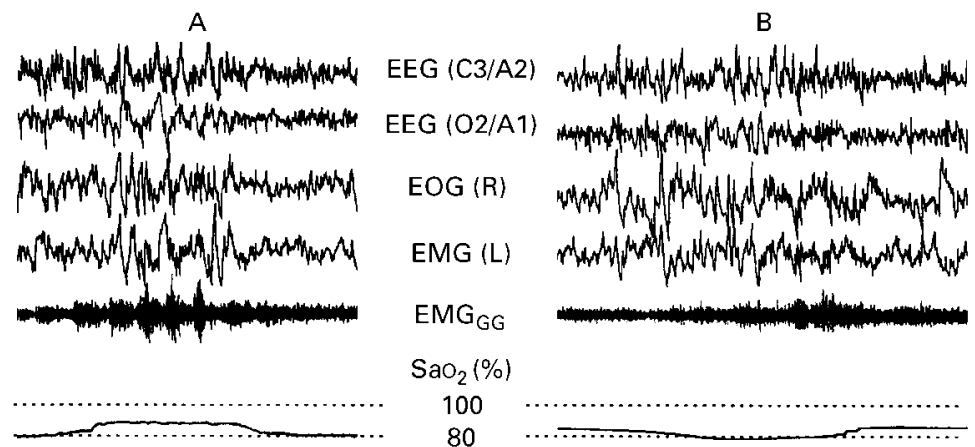

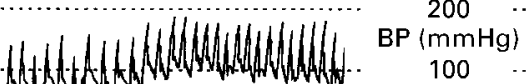

100

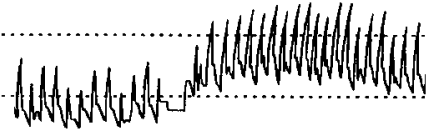

Nasal ventilation
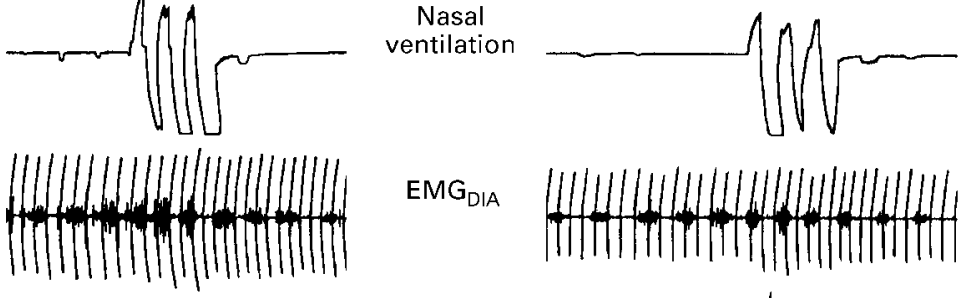

$\mathrm{EMG}_{\mathrm{DIA}}$
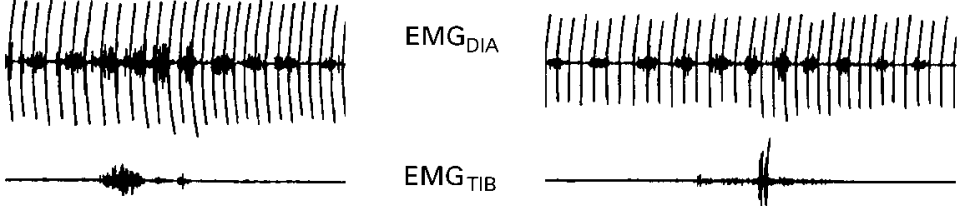

$\mathrm{EMG}_{\mathrm{TIB}}$
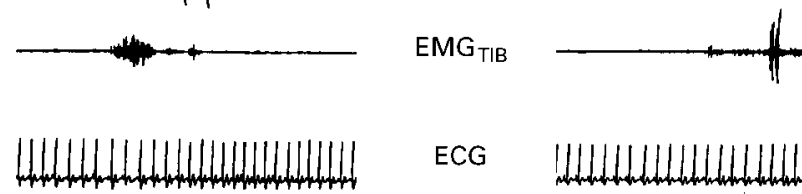

ECG

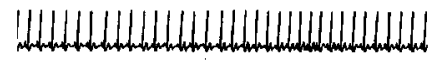

Figure 4 An example of apnoea occurring during (A) the follicular and (B) the luteal phases of the cycle in one subject. The figure shows a significantly potentiated increase in blood pressure following apnoea termination despite similar apnoea length, decrease in $\mathrm{SaO}_{2}$ and level of arousal (as indicated by a similar duration of alpha in the EEG and similar magnitude of genioglossal EMG activity).

non-REM sleep. ${ }^{42}$ During REM sleep a marginal improvement in RDI and decreases in $\mathrm{SaO}_{2}$ have been reported during the luteal phase of the menstrual cycle. ${ }^{42}$ Increased central chemosensitivity associated with the luteal phase of the menstrual cycle is probably responsible for this observed improvement in OSA during this phase. Arousal resulting from respiratory events not meeting standard criteria as outlined by

Rechtschaffen and Kales in $1968^{43}$ (subcriterion events) were not analysed in this study. It may be that, because of increased chemosensitivity during the luteal phase of the menstrual cycle, arousal resulting from obstructive events during sleep may be hastened during the luteal phase as a result of increased sensitivity to blood gas fluctuations. As a result, the length of the obstructive events may not have met the standard criteria of more than 10 seconds to be counted as an obstructive apnoea or hypopnoea. However, considering that a large proportion of the haemodynamic response to obstructive events during sleep is associated with arousal and is independent of changes in blood gas tensions, ${ }^{44}$ these subcriterion events remain a significant risk factor when considering haemodynamic control during sleep apnoea.

\section{Cardiovascular risk in premenopausal women}

While the premenopausal status generally protects against cardiovascular disease, certain populations of women are excluded from this protection-for example, premenopausal diabetic women experience a considerably increased risk of cardiovascular morbidity and mortality compared with healthy premenopausal women. Recent surveys suggest that the incidence of death from cardiovascular disease in diabetic women aged 15-44 (most of whom will be premenopausal) is approximately 19 times that in healthy premenopausal women, while for the same age group in men the risk is approximately 12 times that of a comparable healthy population. ${ }^{45}$ Furthermore, the incidence of cardiovascular disease is similar in premenopausal diabetic women when compared with an age matched male diabetic population. However, in the age group 45-74 (which would include most perimenopausal or postmenopausal women) the risk of cardiovascular death as a ratio of the normal population is similar in men and women (twice the normal population in men and three times the normal population in women).$^{45}$ Thus, previous experience has identified at least one premenopausal group at significant risk of adverse cardiovascular events. Premenopausal women with OSA may be another unidentified subgroup of premenopausal women with a similarly increased risk of developing adverse cardiovascular events.

A recent investigation of haemodynamics during sleep in premenopausal women with apnoea has revealed that the blood pressure profile resulting from apnoea during sleep is similar in men and premenopausal women (fig 3), with a decrease in blood pressure towards the end of the apnoeic period and a surge in blood pressure during the hyperpnoeic phase following arousal. Furthermore, heart rate variability according to the stage of the apnoeic cycle (early apnoea, late apnoea, or hyperpnoeic phase) is similar in men and premenopausal women. ${ }^{42}$

The increase in blood pressure from immediately before apnoea termination to the maximum blood pressure occurring during postapnoea hyperventilation is significantly higher during the luteal phase of ovulatory menstrual 
cycles (fig 4). The increase in the magnitude of the pressor response during the luteal phase is particularly marked during REM sleep, being twice that occurring during the follicular phase. $^{42}$

The haemodynamic responses to obstructive apnoeas during sleep are therefore similar in women to those previously reported in men. However, while there is a slight improvement in sleep disordered breathing during the luteal phase of the menstrual cycle, the haemodynamic consequences of apnoea are significantly amplified during this phase. The potentiated pressor response to obstructive apnoeas in sleep during the luteal phase of the menstrual cycle is most probably mediated by upregulation of peripheral catecholamine receptors.

In conclusion, while mortality data have not been reported specifically in premenopausal women with sleep disordered breathing, increased variability in blood pressure in men with OSA is thought to be associated with their increased cardiovascular risk (stroke, acute myocardial infarction and sudden cardiac death). ${ }^{5}$ Similar cardiovascular variability has been shown to occur in premenopausal women with OSA and, furthermore, this variability is accentuated during the luteal phase of the cycle. We therefore suggest that premenopausal status in these women may not protect against the cardiovascular risk known to accompany OSA in men, and that the existence of hormonal fluctuations associated with the menstrual cycle, far from being protective, may be a confounding factor in cardiovascular risk in this population.

1 Young TB, Palta J, Dempsey J, et al. Occurrence of sleep disordered breathing among middle-aged adults. $N$ Engl $f$ Med 1993;328:1230-5.

2 Young TB, Benes-Malone M, Wever S, et al. Impact of sleep apnoea symptoms and treatment on quality of life. Am $\mathcal{F}$
Respir Crit Care Med 1994;149:A808.

3 Hedner JA, Wilcox I, Sullivan CE. Speculations on the interaction between vascular disease and obstructive sleep apnoea. In: Saunders NA, Sullivan CE, eds. Sleep and breathing. 2nd ed. New York: Marcel Dekker, 1994: 823-46.

4 Hla KM, Young TB, Bidwell T, et al. Sleep apnoea and hypertension. A population-based study. Ann Intern Med hypertension. A

5 Carlson JT, Hedner JA, Ejnell H, et al. High prevalence of hypertension in sleep apnoea patients independent of obesity. Am F Respir Crit Care Med 1994;150:72-7.

6 Andreas S, Hajak G, v.Breska B, et al. Changes in heart rate during obstructive sleep apnoea. Eur Respir f 1992;5:8537.

7 Hedner J, Ejnell H, Sellgren J, et al. Is high and fluctuating muscle sympathetic activity in the sleep apnoea syndrome of pathogenic importance for the development of hypertension? Hypertension 1988;Suppl 6:s529-31.

8 Leuenberger U, Jacob E, Sweer L, et al. Surges of muscle sympathetic nerve activity during obstructive apnoea are linked to hypoxemia. I Appl Physiol 1995;79:581-8.

9 Somers VK, Dyken ME, Clary MP, et al. Sympathetic neural mechanisms in obstructive sleep apnoea. $\mathcal{F}$ Clin Invest ral mechanisms in

10 Somers VK, Mark AL, Abboud FM. Sympathetic activation by hypoxia and hypercapnia: implications for sleep apnoea. Clin Exp Hypertension Part A, Theory \& Practice 1988; 10(Suppl 1):413-22.

11 Jacobsen TN, Morgan BJ, Scherrer U, et al. Relative contributions of cardiopulmonary and sinoaortic baroreflexes in causing sympathetic activation in the human skeletal muscle circulation during orthostatic stress. Circ Res 1993;73 367-78.

12 Somers VK, Dyken ME, Skinner JL. Autonomic and haemodynamic responses and interactions during the Mueller maneuver in humans. 7 Autonom Nerv System 1993;44:253-9.

13 Morgan BJ, Crabtree DC, Puleo DS, et al. Neurocirculatory consequences of abrupt change in sleep state in humans. $\mathcal{F}$ Appl Physiol 1996;80:1627-36.

14 Shimizu T, Takahashi Y, Kogawa S, et al. Muscle sympathetic nerve activity during apneic episodes in sympathetic nerve activity during apneic episodes in
patients with obstructive sleep apnoea syndrome. Electroenpatients with obstructive sleep apnoea syndrom
cephalog Clin Neurophysiol 1994;93:345-52.
15 Carlson JT, Hedner J, Elam M, et al. Augmented resting sympathetic activity in awake patients with obstructive sympathetic activity in awake patients
sleep apnoea. Chest 1993;103:1763-73.

16 Hedner J, Darpo B, Ejnell H, et al. Reduction in sympathetic activity after long-term CPAP treatment in sleep apnoea: cardiovascular implications. Eur Respir f 1995;8:222-9.

17 Shepard JW Jr. Hypertension, cardiac arrhythmias, myocardial infarction, and stroke in relation to obstructive sleep apnoea. Clin Chest Med 1992;13:437-58.

18 Partinen M, Guilleminault C. Daytime sleepiness and vascular morbidity at seven-year follow-up in obstructive sleep apnoea patients. Chest 1990;97:27-32.

19 Pickett CK, Regensteiner JG, Woodard WD, et al. Progestin and oestrogen reduce sleep-disordered breathing in postmenopausal women. f Appl Physiol 1989;66:1656-61.

20 Block AJ. Alcohol ingestion does not cause sleep-disordered breathing in premenopausal women. Alcohol Clin Exp Res 1984;8:397-8.

21 Edwards N, Wilcox I, Polo OJ, et al. Hypercapnic blood pressure response is greater during the luteal phase of the menstrual cycle. F Appl Physiol 1996;81:2142-6.

22 Zwillich CW, Natalino MR, Sutton FD, et al. Effects of progesterone on chemosensitivity in normal men. $f$ Clin Lab Med 1978;92:262-9.

23 White DP, Douglas NJ, Pickett CK, et al. Sexual influence on the control of breathing. F Appl Physiol 1983;54:874-9.

24 Bonekat HW, Dombovy ML, Staats BA. Progesteroneinduced changes in exercise performance and ventilatory response. Med Sci Sports Exerc 1987;19:118-23.

25 Stahl ML, Orr WC, Males L, et al. Progesterone levels and sleep-related breathing during menstrual cycle of normal women. Sleep 1985;8:227-30.

26 Robin ED, Whaley RD, Crump CH. Alveloar gas tensions, pulmonary ventilation and blood $\mathrm{pH}$ during physiologic sleep in normal subjects. $\mathcal{F}$ Clin Invest 1958;37:981-9.

27 Colrain IM, Trinder J, Fraser G. Ventilation during sleep onset in young adult females. Sleep 1990;13:491-501.

28 White DP, Douglas NJ, Pickett CK, et al. Hypoxic ventilatory response during sleep in normal premenopausal women. Am Rev Respir Dis 1982;126:530-3.

29 The Writing Group for the Postmenopausal Oestrogen/ Progestin Interventions (PEPI) Trial. Effects of oestrogen or oestrogen/progestin regimens on heart disease risk factors in postmenopausal women. $\mathscr{f} A M A$ 1995;273:199208.

30 Evans JK, Naish PF, Aber GM. Oestrogen-induced changes in renal haemodynamics in the rat: influence of plasma and intrarenal renin. Clin Sci 1986;71:613-9.

31 Naden RP, Rosenfeld CR. Systemic and uterine responsiveness to angiotensin II and norepinephrine in oestrogentreated nonpregnant sheep. Am f Obstet Gynecol 1985;153: $417-25$

32 Sato N, Miyake S, Akatsu J, et al. Power spectral analysis of heart rate variability in healthy young women during the normal menstrual cycle. Psychosomat Med 1995;57:331-5.

33 Rahman MA, Goodhead K, Medcalf JF, et al. Haemodynamic responses to nonhypotensive central hypovolaemia induced by lower body negative pressure in men and women. Eur f Appl Physiol 1991;63:151-5.

34 Weidner G, Helmig L. Cardiovascular stress reactivity and mood during the menstrual cycle. Women Health 1990;16: $5-21$.

35 Stoney CM, Owens JF, Matthews KA, et al. Influences of the normal menstrual cycle on physiologic functioning during behavioral stress. Psychophysiology 1990;27:125-35.

36 Karpanou EA, Vyssoulis GP, Georgoudi DG, et al. Ambulatory blood pressure changes in the menstrual cycle of hypertensive women. Significance of plasma renin activity values. Am f Hypertens 1993;6:654-9.

37 Tersman Z, Collins A, Eneroth P. Cardiovascular responses to psychological and physiological stressors during the menstrual cycle. Psychosom Med 1991;53:185-97.

38 Mehta V, Chakrabarty AS. Autonomic functions during different phases of menstrual cycle. Ind $\mathcal{F}$ Physiol Pharmacol 1993;37:56-8

39 Tollan A, Oian P, Kjeldsen SE, et al. Progesterone reduces sympathetic tone without changing blood pressure or fluid balance in men. Gynecol Obstet Invest 1993;36:234-8.

40 Re M, Gemelli A, Falcone M, et al. Cardiovascular effects induced by progesterone administration. Panminerva Med 1989;31:28-9.

41 Cistulli PA, Barnes DJ, Grunstein RR, et al. Effect of shortterm hormone replacement in the treatment of obstructive sleep apnoea in postmenopausal women. Thorax 1994;49: 699-702.

42 Edwards N, Wilcox I, Sullivan CE. Influence of the menstrual cycle on haemodynamic responses in obstructive sleep apnoea. 13th Congress, European Sleep Research Society, 1996

43 Rechtschaffen A, Kales A. A manual of standardized terminnology, techniques and scoring systems for sleep stages of human subjects. Washington, DC: National Institutes of Health, 1968.

44 Ringler J, Basner RC, Shannon R, et al. Hypoxemia alone does not explain blood pressure elevations after obstructive apnoeas. F Appl Physiol 1990;69:2143-8.

45 Reckless JP. The epidemiology of heart disease in diabetes mellitus. In: Taylor KG, eds. Diabetes and the heart. Kent: Castle House Publications, 1987: 1-18. 